\title{
Neural activity, neural connectivity, and the processing of emotionally valenced information in older adults: links with life satisfaction
}

\author{
Robert J. Waldinger • Elizabeth A. Kensinger • \\ Marc S. Schulz
}

Published online: 18 May 2011

(C) Psychonomic Society, Inc. 2011

\begin{abstract}
This study examines whether differences in latelife well-being are linked to how older adults encode emotionally valenced information. Using fMRI with 39 older adults varying in life satisfaction, we examined how viewing positive and negative images would affect activation and connectivity of an emotion-processing network. Participants engaged most regions within this network more robustly for positive than for negative images, but within the PFC this effect was moderated by life satisfaction, with individuals higher in satisfaction showing lower levels of activity during the processing of positive images. Participants high in satisfaction showed stronger correlations among network regions - particularly between the amygdala and other emotion processing regions - when viewing positive, as compared with negative, images. Participants low in satisfaction showed no valence effect. Findings suggest that late-life satisfaction is linked with how emotion-processing regions are engaged and connected during processing of valenced information. This first demonstration of a link between neural recruitment and late-life well-being suggests that differences
\end{abstract}

The study was supported by a grant from the National Institute on Aging (R01 AG 034554).

R. J. Waldinger $(\square)$

Department of Psychiatry,

Massachusetts General Hospital and Harvard Medical School,

15 Parkman Street - WACC 812,

Boston, MA 02114, USA

e-mail: rwaldinger@partners.org

E. A. Kensinger

Department of Psychology, Boston College,

Chestnut Hill, MA, USA

M. S. Schulz

Department of Psychology, Bryn Mawr College,

Bryn Mawr, PA, USA in neural network activation and connectivity may account for the preferential encoding of positive information seen in some older adults.

Keywords Aging $\cdot$ Well-being $\cdot$ Life satisfaction $\cdot$ Neural connectivity $\cdot$ Positivity effect

\section{Introduction}

Aging is typically characterized as a process of uniform decline across mental and physical domains. Yet emotional well-being has been found to improve with age (Mather \& Carstensen, 2005; Mroczek \& Kolarz, 1998; Williams et al., 2006). This improvement is particularly important because of strong links between emotion in the elderly and physical and cognitive health (Benyamini, Idler, Leventhal, \& Leventhal, 2000; Hawkley \& Cacioppo, 2007). In recent efforts to understand the sources of emotional well-being in the elderly, investigators have studied the ways in which emotionally valenced information is processed when initially encountered (Mather \& Carstensen, 2005; Murphy \& Isaacowitz, 2008).

Research demonstrates that older adults process emotionally valenced information differently than do younger adults. In contrast to younger adults, older adults manifest a positivity effect - that is, preferential attention to and memory for positively valenced over negatively valenced information (Mather \& Carstensen, 2005). Age differences in emotion processing are found at a neural level in the degree to which specific brain regions respond to the input of emotionally valenced information. In particular, the amygdala in older adults has been shown to respond less to negative than to positive information (e.g., Mather et al., 2004; St. Jacques, Bessette-Symons, \& Cabeza, 2009). 
Although the role of the amygdala in emotion processing was initially believed to be restricted to fear processing or negative emotions (e.g., Kjelstrup et al., 2002; Öhman \& Mineka, 2001), recent work has demonstrated amygdala responses to positive as well as negative emotions (Hamann, Ely, Hoffman, \& Kilts, 2002) and has led to more general hypotheses about the role of the amygdala in emotion processing. For instance, Sander, Grafman, and Zalla (2003) have posited that the amygdala serves more generally as a relevance detector, rather than being specifically linked with negative emotion processing. Relatedly, Davis and Whalen (2001) proposed that the amygdala may be activated in situations where people are particularly vigilant, regardless of whether the increased attention is directed toward aversive or appetitive stimuli. If the amygdala plays a more general role as a salience detector, or if its activity is modulated by the vigilance accorded to a stimulus, this raises the possibility that older adults' decreased amygdala response to negative information and their relatively preserved response to positive stimuli could be related to differences in perceptions of the relevance of positive and negative stimuli.

Differences in amygdala engagement in relation to stimulus salience may account for the finding that amygdala response to negative versus positive information changes as a function of age. Moreover, the degree to which the amygdala distinguishes the two valences may differ within a single age group on the basis of individual differences in the perceived salience of emotionally valenced stimuli. The present study addressed the latter possibility by using fMRI to examine whether differences in late-life well-being are linked to activation of amygdala-mediated networks in response to positive and negative information.

Although most prior studies examining brain responses to emotionally valenced information in the elderly have focused on activation of particular brain regions-most commonly, the amygdala (e.g., Mather et al., 2004; Wright, Wedig, Williams, Rauch, \& Albert, 2006) - it is clear that the amygdala acts in concert with other regions such as the orbital frontal cortex and the ventromedial frontal cortex to ascertain the meaning of an emotional event (Hartley \& Phelps, 2010; Ochsner, Bunge, Gross, \& Gabrieli, 2002; Phelps \& LeDoux, 2005). Connectivity among these brain regions is likely to be important for understanding older adults" "positivity effect." For example, older adults have stronger connectivity among the ventromedial prefrontal cortex, amygdala, and hippocampus than do young adults during the processing of positive information (Addis, Leclerc, Muscatell, \& Kensinger, 2010), and older adults can shower weaker connections than do young adults between the amygdala and the hippocampus during the encoding of negative information (St. Jacques, Dolcos, \& Cabeza, 2009).
Age-related patterns of emotion processing have often been hypothesized to be connected to levels of well-being (e.g., Charles \& Carstensen, 2010). Yet there has not been an empirical demonstration of a link between older adults' emotional well-being and their recruitment of emotionprocessing circuitry. The present study directly examined whether this link could be revealed. We examined both activity and connectivity within regions previously revealed to be an important part of an emotional memory network (Addis et al., 2010; also see the meta-analysis by Murty, Ritchey, Adcock, \& LaBar, 2011), focusing on how participants' reported satisfaction with life was related to neural activity or connectivity as they processed positively valenced and negatively valenced visual images. In addition to the amygdala, this network of regions included the ventromedial and orbitofrontal cortex, which have been associated with attention toward internal states (including affective ones) and with the regulation of those states (e.g., Cooney, Joorman, Atlas, Eugene, \& Gotlib 2007; Kalisch, Wiech, Critchley, \& Dolan, 2006); the hippocampus, known to be critical for the successful storage of information in memory (e.g., Cipolotti \& Bird, 2006; Spaniol et al., 2009); the thalamus, which can serve as an interface between the prefrontal and hippocampal regions (e.g., Vertes, 2006); and the fusiform gyrus, a part of the ventral visual processing stream (Ungerleider \& Haxby, 1994) whose processing can be modulated by the presence of emotion (e.g., Padmala \& Pessoa, 2008). Each of these regions has been shown to interact extensively with the amygdala. For instance, prefrontal interactions with the amygdala often guide emotion regulatory processes (e.g., Cooney et al., 2007; Kalisch et al., 2006), hippocampal interactions with the amygdala intensify memory encoding and consolidation (e.g., Hamann, 2001), and fusiform interactions with the amygdala augment visual attention and sensory processing (e.g., Padmala \& Pessoa, 2008).

The present study addressed the hypothesis that within a cohort of older adults, amygdala connectivity with the other regions within this network would differ as a function of life satisfaction. Such a relationship between amygdala connectivity and life satisfaction could be interpreted in two distinct ways: (1) that well-being is shaped by the degree to which positive versus negative stimuli are interpreted as salient or personally relevant (Carstensen \& Mikels, 2005), or (2) that patterns of amygdala-mediated neural connectivity shape well-being.

A critical first step is to establish whether or not such a link exists. The present study is one of the first to examine patterns of connectivity among brain regions that might be linked with emotional well-being in older adults (see also Addis et al., 2010; St. Jacques, Dolcos, \& Cabeza, 2009), and it is the first to examine individual differences within a cohort of older adults in neural recruitment during emotion 
processing. Our aim was to examine whether different levels of emotional well-being are associated with different patterns of neural connectivity when processing positively and negatively valenced information. We predicted that those higher in life satisfaction would show stronger connectivity between the amygdala and the other regions of the emotion-processing network during the encoding of positively valenced information, as compared with negatively valenced information; this finding would be consistent with a connection between higher life satisfaction and higher salience of positive information. To address the relation between life satisfaction and neural activity and connectivity, we focus on a sample of older adults, because of previous findings of changes in emotion processing in this age group (e.g., Carstensen, Pasupathi, Mayr, \& Nesselroade, 2000) and hypothesized links between these changes and well-being (e.g., Löckenhoff \& Carstensen, 2004).

\section{Method}

\section{Participants}

Participants enrolled in a longitudinal study of adult development (Glueck \& Glueck, 1968; Heath, 1945; Vaillant, 1977) were contacted if they met eligibility criteria for fMRI. Among the 59 participants who agreed to participate in an fMRI scan assessing emotion processing, usable data was obtained from 39 participants (10 females, 29 males; mean age 79.5 years, range $64-89, S D=6.2$; mean years of education 15.1 , range $10-20, S D=2.9$ ). The remaining participants were excluded due to scanner malfunction (2 participants), projector malfunction (2), poor task performance (3), excessive head motion (4), or vision problems that prevented viewing of the images ( 9 participants). Thirty-five participants were right-handed or ambidextrous, and 4 were left-handed. Of the 4 left-handed participants, 3 were in the high-life-satisfaction group, and 1 was in the low-satisfaction group.

\section{Measures}

Life satisfaction was measured at the time of the MRI procedure using the Satisfaction With Life Scale (SWLS; Diener, Emmons, Larsen, \& Griffin, 1985), a widely-used self-report questionnaire that is designed to measure cognitive-evaluative aspects of subjective well-being. Participants rate how much they agree or disagree with five life satisfaction statements on 7-point Likert-type scales. Scores are summed to generate a total score ranging from 5 to 35 . The SWLS has been shown to have good reliability and validity (Pavot, Diener, Colvin, \&
Sandvik, 1991). Past research has found sizable correlations between the SWLS and measures of positive affect (e.g., $r=.55$; Palmer, Donaldson, \& Stough, 2002), negative affect (e.g., $r=-.57$; Chang, Watkins, \& Banks, 2004), and depression (e.g., $r=-.72$; Blais, Vallerand, Pelletier, \& Briere, 1989).

\section{MRI methods}

Structural and functional magnetic resonance images were acquired on a General Electric MRI scanner (General Electric Medical Systems, Milwaukee, WI). Anatomic images were acquired with a multiplanar rapidly acquired gradient echo (MP-RAGE) sequence. Functional images were collected using a gradient echo, echoplanar imaging (EPI) pulse sequence sensitive to blood oxygenation level dependent (BOLD) signal (repetition time $=3,000 \mathrm{~ms}$, effective echo time $=$ $40 \mathrm{~ms}$, flip angle $=90^{\circ}$ ). Twenty-seven axial-oblique slices (3.12-mm thickness) were aligned along the line between the anterior commissure and the posterior commissure and were acquired in an interleaved fashion. This slice acquisition allowed for coverage of most of the brain, although the dorsal-most aspects of the frontal and parietal lobes were not included within the field of view.

During the fMRI scan, participants viewed 180 pictures (60 positive and arousing, 60 negative and arousing, 60 neutral) selected from the IAPS database (Lang, Bradley, \& Cuthbert, 1999). Normative data for these pictures had previously been gathered to ensure that older adults' ratings agreed with the IAPS norms (as reported in Leclerc \& Kensinger, 2008). Each picture was presented for $3 \mathrm{~s}$. Pictures from the different emotion categories were pseudorandomly intermixed, with interstimulus intervals ranging from 3 to $12 \mathrm{~s}$, in order to create the jitter necessary to optimize detection of the hemodynamic response associated with each picture (Dale, 1999). Participants were asked to view the series of pictures as if they were watching TV and to press a button each time the picture changed. Outside of the scanner, participants performed a surprise recognition memory task. Only items that were correctly recognized were included in fMRI analyses, because for these items it was clear that the participants had attended and processed the image while in the MRI scanner.

Preprocessing and data analysis were conducted within SPM2 (Wellcome Department of Cognitive Neurology). Standard preprocessing included slice-timing correction, rigid body motion correction, normalization to the Montreal Neurological Institute template (resampling at $3 \mathrm{~mm}$ cubic voxels), and spatial smoothing (using an 8-mm full-width half-maximum isotropic Gaussian kernel). 
Data analysis

Region definition We used a region-of-interest (ROI) approach for all analyses. ROIs were taken from the coordinates from a prior study examining age-related changes in emotion processing (Addis et al., 2010). This selection of ROIs was chosen because we were concerned that using group analyses from the present dataset to define regions could allow a subset of our participants (e.g., those high in life satisfaction) to bias region selection. By using coordinates from a prior study, we ensured that the region selection was unbiased with regard to the individual differences present in our present sample.

For each of the ROIs identified in the Addis et al. (2010) study (except for the dorsomedial PFC, which could not be imaged in our study due to our slice acquisition parameters), we defined 8-mm spheres centered around the maximum voxel reported in that article (see Table 1 for regions and coordinates). Within each of these ROIs, a hemodynamic response function was calculated for each individual participant and for each valence type (positive, negative, neutral) as a function of peristimulus time (0-21 s). The average percentage of signal change reached within peristimulus times 3-9 s was then computed for each participant, and these signal change values underwent further analyses.

Regional activity We first examined the activity within each ROI, using an ANOVA with the within-subjects factor of valence (positive, negative, neutral) to test for differences. We included scores on the satisfaction with life scale as a covariate; gender and age were also included as covariates in these analyses. These analyses allowed us to examine whether life satisfaction moderated the pattern of activity within the ROIs.

Regional connectivity After examining the activity within each separate ROI, we then examined the connectivity among the regions by computing correlation matrices and using structural equation modeling (SEM) analyses.

Table 1 Regions included in the anatomical model*

\begin{tabular}{lll}
\hline Region & Hemisphere & Coordinates \\
\hline Amygdala & $\mathrm{L}$ & $-28,-3,-12$ \\
Fusiform & $\mathrm{L}$ & $-46,-48,-18$ \\
Hippocampus & $\mathrm{L}$ & $-36,-7,-23$ \\
Orbitofrontal cortex & $\mathrm{L}$ & $-36,44,-8$ \\
Thalamus & $\mathrm{L}$ & $-6,-20,-2$ \\
Ventromedial prefrontal cortex & Bilateral & $0,39,-5$
\end{tabular}

* Regions were selected because of their known contributions to the emotional memory network (Addis et al., 2010).
All SEM calculations were performed using Lisrel 8.30 (Joreskog \& Sorbom, 1993). These analyses require the definition of an anatomical and a functional model (McIntosh \& Gonzalez-Lima, 1994). For the anatomical model, we included all of the ROIs listed in Table 1 (from Addis et al., 2010), and we included connections that were both plausible anatomically and also of interest to our hypotheses regarding emotion processing. For all projections except for those involving the fusiform, we modeled bidirectional connections. For the fusiform, we focused on the feed-forward connections because we were interested in how visual information was transmitted to emotionprocessing regions.

For the functional model, participants were divided into two groups on the basis of a median split of scores for life satisfaction. Although we recognize that the use of a median split has limitations, SEM has yet to be used with fMRI data while including a continuous-variable moderator. Therefore, performing the median split on our participant groups allowed us to employ an existing, well-validated method for using SEM with fMRI data (McIntosh \& Gonzalez-Lima, 1994). Pairing these SEM analyses with the analyses of regional activity that treated life satisfaction as a continuous moderator allowed us to address some of the limitations of depending solely on a median split.

Separate functional models were examined for the highsatisfaction group and for the low-satisfaction group. For each group, correlation matrices were calculated separately for each valence (positive and negative), revealing the correlation of percentage of signal change between each of the ROIs within the anatomical model (see Table 2 for correlation matrices). Because no region included in the model was shown to activate significantly during the processing of neutral images (see Fig. 1), SEMs were not computed for the neutral images. For each of the two SEMs (one comparing negative with positive stimuli in the highsatisfaction group, and the other comparing negative with positive stimuli in the low-satisfaction group), estimates of path coefficients - indicating strength and direction of influence - were calculated on the basis of these correlation matrices.

To determine whether there were significant differences in connectivity between the conditions (i.e., between negative and positive valence), we used a stacked-model SEM approach (McIntosh \& Gonzalez-Lima, 1994) to compare a null model, in which the path coefficients for positive and negative valence were assumed to be equal, with an alternate model in which the path coefficients for negative and positive valence were allowed to vary. The fits of the null and alternate models were determined using a goodness-of-fit $\chi^{2}$ test, and the differences between the models were calculated by comparing these goodness-of-fit $\chi^{2}$ values to obtain a $\chi_{\text {diff }}^{2}$. If the $\chi_{\text {diff }}^{2}$ between models 
Table 2 Correlation matrices for the six ROIs as a function of image valence and life satisfaction group

\begin{tabular}{|c|c|c|c|c|c|c|}
\hline \multicolumn{7}{|c|}{$\begin{array}{l}\text { Low Satisfaction Group }(\mathrm{N}=18) \text { below } \\
\text { diagonal }\end{array}$} \\
\hline \multicolumn{7}{|c|}{ Negative Images } \\
\hline & Fusiform & OFC & Hippocampus & Amygdala & Thalamus & vmPFC \\
\hline Fusiform & -- & 0.37 & 0.36 & $\underline{-0.26}$ & $\underline{-0.25}$ & 0.08 \\
\hline $\mathrm{OFC}$ & 0.36 & -- & -0.16 & $\overline{0.19}$ & $\overline{0.16}$ & $0.61 * *$ \\
\hline Hippocampus & 0.28 & 0.34 & -- & -0.14 & -0.27 & -0.04 \\
\hline Amygdala & $\underline{0.33}$ & $0.64 * *$ & $\underline{0.75}^{* *}$ & -- & $\underline{0.44 *}$ & 0.32 \\
\hline Thalamus & $\underline{0.52 *}$ & $0.53 *$ & $\underline{0.74} * *$ & $\underline{0.79} * *$ & -- & $\underline{0.40^{*}}$ \\
\hline vmPFC & -0.20 & 0.23 & 0.02 & 0.05 & $\underline{-0.25}$ & -- \\
\hline \multicolumn{7}{|c|}{ Positive Images } \\
\hline Fusiform & -- & $0.83 * *$ & $0.73 * *$ & $0.64 * *$ & $0.77 * *$ & $0.63 * *$ \\
\hline OFC & $\underline{0.43}$ & -- & $0.58 * *$ & $0.54 *$ & $0.77 * *$ & $0.64 * *$ \\
\hline Hippocampus & 0.39 & $0.60 * *$ & -- & $0.89 * *$ & $0.72 * *$ & $0.66 * *$ \\
\hline Amygdala & 0.42 & $0.69 * *$ & $0.89 * *$ & -- & $0.78 * *$ & $0.69 * *$ \\
\hline Thalamus & $0.59 *$ & 0.46 & $0.87 * *$ & $0.86^{* *}$ & -- & $0.77 * *$ \\
\hline vmPFC & 0.42 & $0.60 * *$ & $0.74 * *$ & $0.66 * *$ & $0.74 * *$ & -- \\
\hline \multicolumn{7}{|c|}{ Neutral Images } \\
\hline Fusiform & -- & -0.09 & 0.37 & 0.12 & 0.08 & 0.08 \\
\hline $\mathrm{OFC}$ & 0.39 & -- & 0.35 & $0.51 *$ & $\underline{0.36}$ & $\underline{0.51 *}$ \\
\hline Hippocampus & 0.38 & $0.66 * *$ & -- & $0.67 * *$ & $0.65 * *$ & $0.51 *$ \\
\hline Amygdala & 0.17 & $0.63 * *$ & $0.83^{* *}$ & -- & $0.78 * *$ & $0.79 * *$ \\
\hline Thalamus & 0.46 & $\underline{0.88 * *}$ & $0.78 * *$ & $0.73 * *$ & -- & $0.87 * *$ \\
\hline vmPFC & 0.20 & $\underline{0.86^{* *}}$ & $0.64 * *$ & $0.63 * *$ & $0.77 * *$ & -- \\
\hline
\end{tabular}

Underlined values denote a significant difference $(\mathrm{p}<.05)$ in correlation strength between low- and high-satisfaction individuals. Asterisks $(*)$ denote correlations that are significantly different from zero $(* \mathrm{p}<.05 ; * * \mathrm{p}<.01)$. Boldface type and italics denote correlations that are significantly different $(\mathrm{p}<.05)$ within groups across conditions. These comparisons were made only between positive and negative images. The approach described by Steiger (1980) and Kashy and Snyder (1995) was used to compare correlations within persons across conditions.

reached statistical significance $(p<.05)$, that indicated that there were significant differences in the connections within the emotion-processing network as a function of stimulus valence. If the $\chi_{\text {diff }}^{2}$ between models did not reach significance, the null model could not be rejected, suggesting that the same neural connectivity was present when processing both positive and negative images. If the null and alternate models were found to differ significantly, additional SEM analyses were conducted to determine which connections

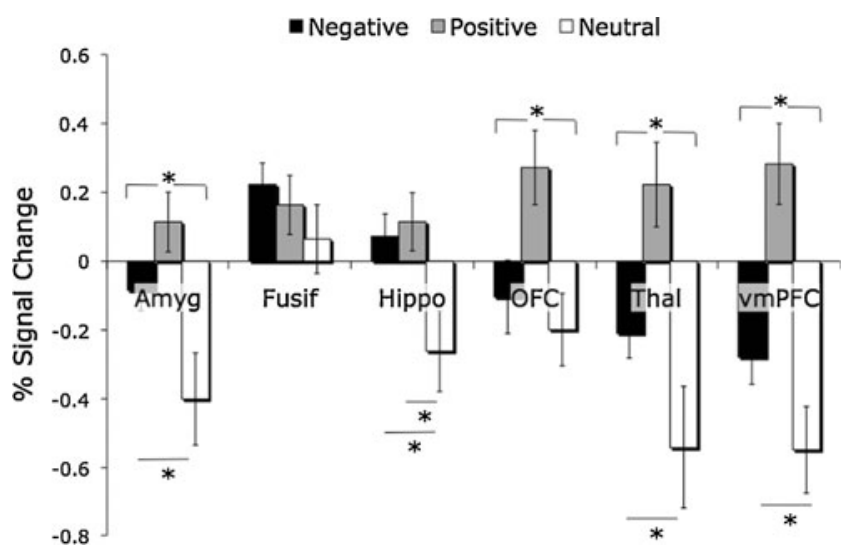

Fig. 1 Signal change in the six regions of interest (ROIs) as a function of valence (ROI coordinates listed in Table 1$)$. *Significant difference, $p<.05$ contributed significantly to the differences. In these analyses, each connection was allowed to vary in a stepwise manner. If allowing a connection to vary caused a reduction in the $p$-value associated with the $\chi_{\text {diff }}^{2}$, this connection was allowed to vary in the final model. Otherwise, the strength of the connection remained fixed in the final model.

Because the order in which connections are allowed to vary can influence whether a connection is deemed to have a significant influence on the fit of the model, we conducted a series of different stepwise removals, using a different path removal order each time. The model reported here is the model that led to the largest $\chi_{\text {diff }}^{2}$ from the null model while still retaining good model stability (as described in Addis et al., 2010) and no beta-weights reported above a value of 1 .

\section{Results}

The mean SWLS score in this sample was 27.0 (range 11-35, $\mathrm{SD}=6.5)$, similar to the mean score $(25.8)$ in a group of 53 elderly adults (average age 75) reported by Diener and colleagues (SWLS; Diener, Emmons, Larsen, \& Griffin, 1985). Using a median split, participants were categorized as high or low on life satisfaction; those categorized as high in life satisfaction (21 participants) had scores of 29 and above, while the other 18 participants were categorized as low in life satisfaction. Participants in the high- and low- 
satisfaction groups did not differ significantly with respect to age, years of education, IQ (as measured by the AMNART; Blair \& Spreen, 1989), or cognitive functioning (CERAD memory and executive functioning composites; Morris et al., 1989). Information about self-rated physical health was available for 27 of the 29 men in the sample; self-reported health (on average, 16 months prior to the MRI) did not differ across high- and low-satisfaction groups for these men.

Neural activity during processing of emotionally valenced stimuli

ROI analyses first examined the pattern activity in each of the six regions as a function of valence. Separate ANOVAs were conducted for the signal change within each region, with emotional valence (positive, negative, neutral) as a within-subjects factor. For the fusiform gyrus, this ANOVA revealed no significant effect of valence, $F(2,37)=1.27, p>.25$, partial eta-squared $\left(\eta_{\mathrm{p}}^{2}\right)=.076$. For the hippocampus, the ANOVA revealed a significant effect of valence, $F(2,37)=3.67, p<.05$, $\eta_{\mathrm{p}}^{2}=.17$, with signal change enhanced for positive or negative pictures, as compared with neutral ones, $p<.05$, but not differing significantly between positive and negative pictures, $p>$.6. For the amygdala, OFC, thalamus, and vmPFC, all ANOVAs revealed significant effects of valence, all $F_{\mathrm{s}}(2,37)>5.9$, all $p \mathrm{~s}<.01$, all $\eta_{\mathrm{p}}^{2} \mathrm{~s}>.24$. In each of these regions, signal change was greater for positive pictures than for negative or neutral ones, $p<.05$. In the amygdala, thalamus, and vmPFC, signal change also was greater for negative items than for neutral, $p<.05$ (see Fig. 1).

Because we were particularly interested in how individual differences in life satisfaction could impact this pattern of neural activity, we reran the ANOVAs including life satisfaction scores, gender, and age as covariates. These analyses indicated that life satisfaction scores moderated the effect of emotion on activity only in the $\mathrm{OFC}, F(2,34)=3.42, p<.05$, $\eta_{\mathrm{p}}^{2}=.17$, and in the vmPFC, $F(2,34)=5.38, p<.01$, $\eta_{\mathrm{p}}^{2}=.17$. In all other regions, the moderation was not significant, $F<2, p>.15$. Neither age nor gender was a significant moderator in any of the ANOVAs $(p>.15)$.

To further clarify the nature of the moderating impact of life satisfaction, univariate ANOVAs were run separately for each valence of information processed within the OFC or the vmPFC. These ANOVAs revealed that life satisfaction scores influenced the activity in the OFC, $F(1,38)=5.49, p<.05$, and vmPFC, $F(1,38)=8.76$, $p<.01$, during the processing of positive images but did not influence the activity in either region during the processing of negative or neutral images (all $p s>.1$ ). In both of these regions, higher levels of life satisfaction were associated with lower activity during the processing of positive information.
Neural connectivity during processing of emotionally valenced stimuli

Having established that life satisfaction was linked to activity within PFC regions, we then examined whether it also was associated with the connectivity within a network of emotion-processing regions. Inspection of the correlation matrices for the two groups (see Table 2) pointed to substantial between-group differences in connectivity: The high-satisfaction group had mostly negative correlations among the different ROIs when processing negative images but had strong positive correlations among the regions when processing positive images.

There did not appear to be as large a discrepancy in the strength of the regional correlations for the low-satisfaction group, with correlations being of moderate positive strength regardless of the valence of image being processed. Fisher $z$-tests confirmed that nearly all correlations were significantly stronger during the encoding of the positive, as compared with the negative, images in the high-satisfaction individuals, whereas in the low-satisfaction individuals, only the correlations with the vmPFC differed based on the valence (positive vs. negative) of the images being encoded. Between-group comparisons of the correlation coefficients (using Fisher $z$-tests) further confirmed these differences: High-satisfaction individuals had consistently lower correlations during the processing of negative images than did low-satisfaction individuals (significant group differences denoted by underlining in Table 2).

High-satisfaction individuals also showed a few instances of correlations lower than those shown by lowsatisfaction individuals during the processing of neutral images. By contrast, all correlations that differed between the two groups for the positive images were in the opposite direction, with high-satisfaction individuals showing stronger correlations than did low-satisfaction individuals. Thus, it is only for the positive images that high-satisfaction individuals show stronger correlations between regions than do low-satisfaction individuals.

SEM analyses allowed us to examine further whether the effect of valence on neural connectivity was different in those high versus low in life satisfaction. The omnibus SEM analysis for the high-satisfaction group revealed a significant effect of valence on the effective connectivity within the emotion-processing network; the alternate model was a significantly better fit than the null model, $\chi_{\text {diff }}^{2}(15)=55.5, p<.001$. A stepwise assessment of the connections that differed significantly across conditions revealed that a number of connections were stronger during the encoding of positive pictures, as compared with negative ones. These connections included projections from the amygdala to the orbitofrontal cortex, the ventromedial prefrontal cortex, and the thalamus, as well as projections 
from the fusiform to the hippocampus and from the hippocampus to the amygdala (see Fig. 2).

The omnibus SEM analysis for the low-satisfaction group revealed no significant effect of valence on the effective connectivity within the emotion processing network, $\chi_{\text {diff }}^{2}(15)=22.2, p>.10$. In the low-satisfaction group, the amygdala showed strong projections to prefrontal and thalamic regions, and the strength of these connections did not differ on the basis of the valence of the information being processed (Fig. 3).

Comparisons of the correlation strengths confirm that in the high-satisfaction group, all but one correlation coefficient differed by at least .34 (and most differed by more than .5), whereas in the low-satisfaction group, with the exception of five correlations (four of which were with the vmPFC), correlation coefficients differed by less than .26 . Thus, only for those high in satisfaction do the majority of connections within the emotion-processing network differ for positively and negatively valenced items; for the low-satisfaction group, the network appears to be engaged similarly regardless of the valence of information being processed.

\section{Discussion}

This study is the first to demonstrate links between late-life well-being and patterns of activity and connectivity within a neural network involved in emotion processing. Life satisfaction was a moderator of activity within the OFC and vmPFC, and only older adults high in life satisfaction showed stronger connectivity within an amygdala-mediated network in response to positive visual images, as compared with negative ones, whereas no such valence effect was seen in older adults who reported lower life satisfaction.

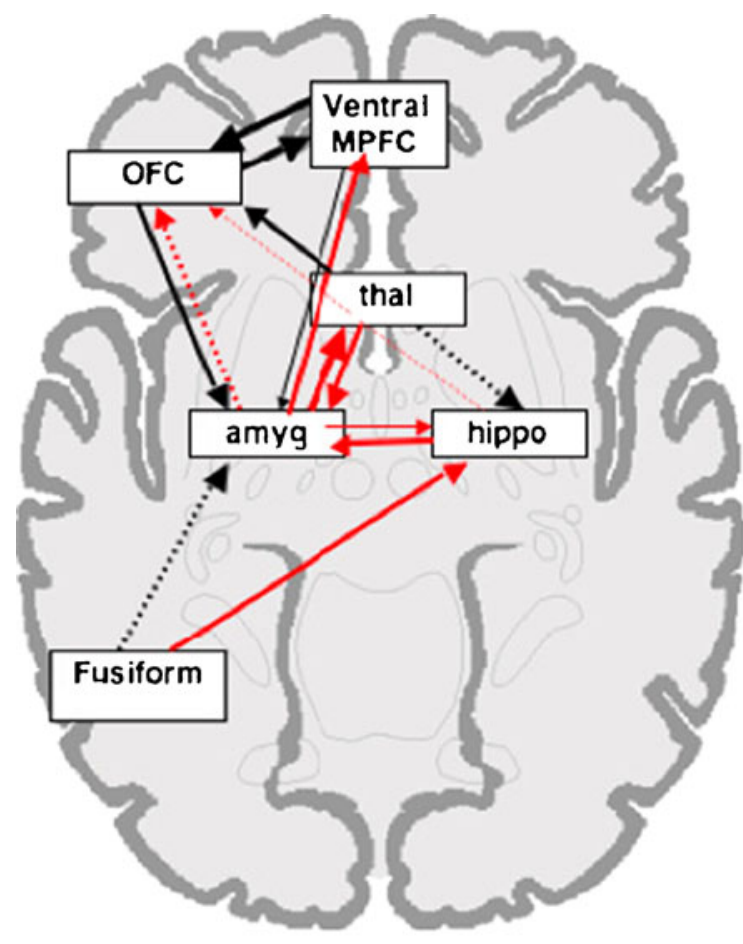

Processing of Negative Pictures Later Remembered

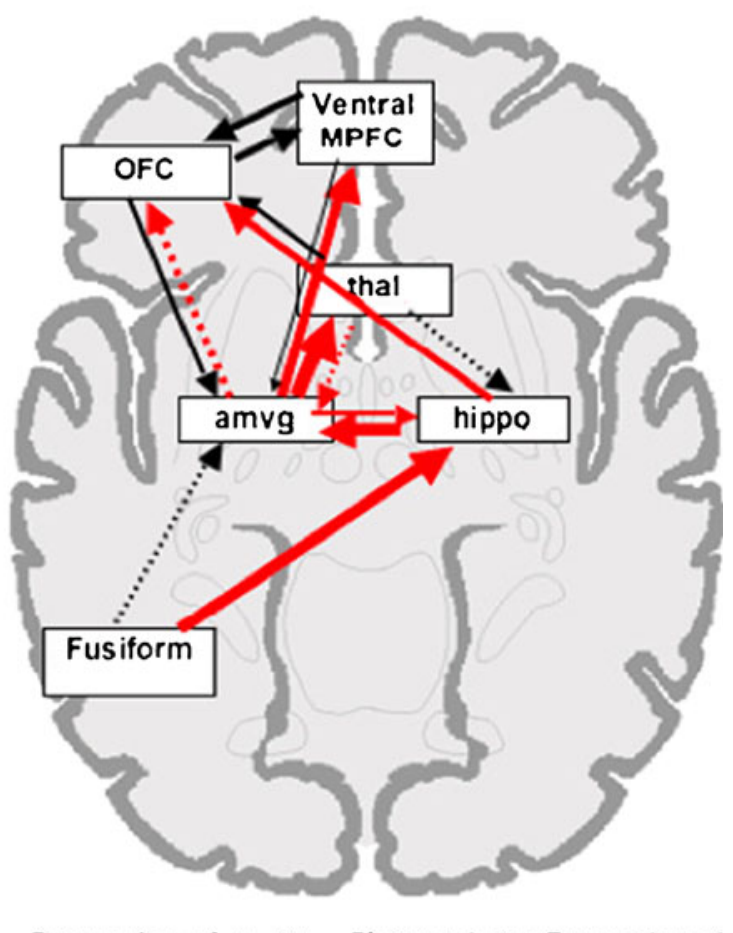

Processing of Positive Pictures Later Remembered

Positive connections

\section{Negative connections}

$$
\begin{aligned}
& .01-0.1 \\
& 0.1-0.3 \\
& 0.3-0.5 \\
& 0.5-0.7 \\
& 0.7-1.0
\end{aligned}
$$

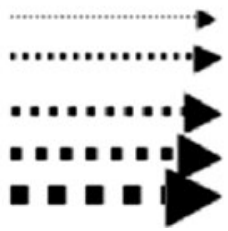

Fig. 2 Patterns of effective connectivity for the high-satisfaction group during encoding of negative pictures (at left) and positive pictures (at right). Red lines denote connections of significantly different strength for negative and positive pictures; black lines signify connections of similar strength for each valence. All pathways included in the anatomical model are depicted 


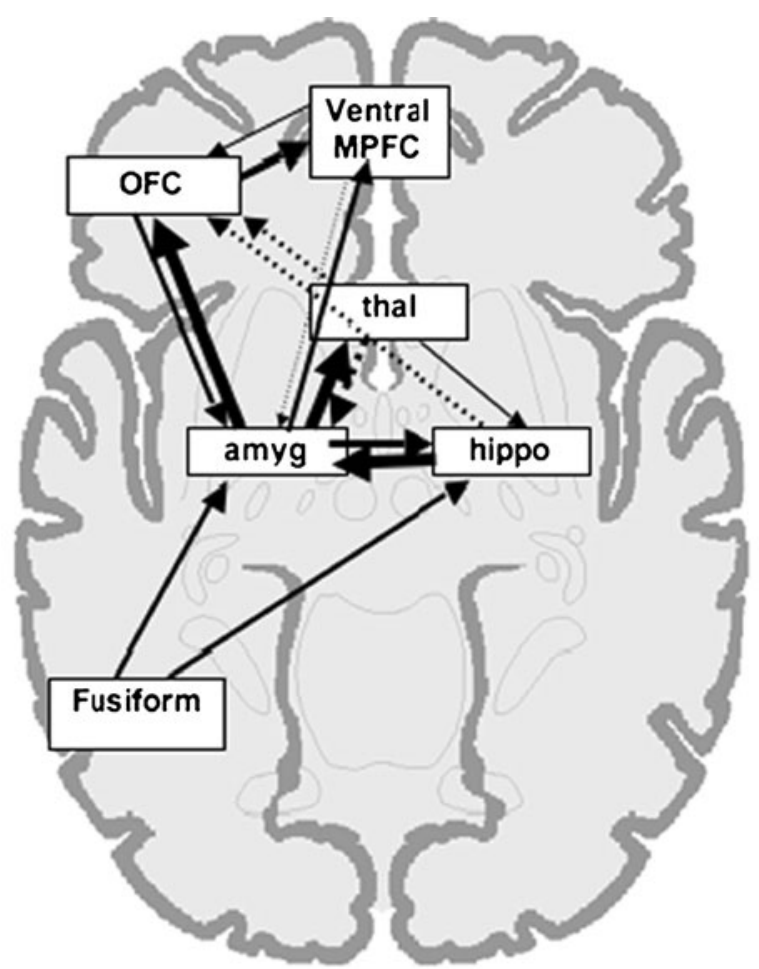

Processing of Negative or Positive Pictures Later Remembered

Positive connections

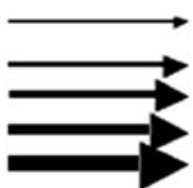

$$
\begin{aligned}
& .01-0.1 \\
& 0.1-0.3 \\
& 0.3-0.5 \\
& 0.5-0.7 \\
& 0.7-1.0
\end{aligned}
$$

Negative connections

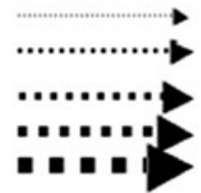

Fig. 3 Patterns of effective connectivity for the low-satisfaction group during encoding of positive and negative pictures (no significant differences between patterns for positive and negative pictures). All pathways included in the anatomical model are depicted

The study adds to the literature on the neural underpinnings of emotion processing in older adults in two ways. First, prior studies of emotion processing and aging have compared older with younger adults but have not examined differences among older adults based on differences in subjective well-being. Second, prior studies of neural activation in response to emotionally valenced stimuli have focused on particular areas of the brain but have not examined connectivity of brain regions associated with emotion processing.

The preferential attention to and memory for positive information seen in many older adults has been associated with differences in amygdala activation (e.g., Mather et al., 2004). This linkage, in turn, has been proposed to be associated with greater emotional well-being, yet this study is the first to demonstrate a direct link between amygdala connectivity and subjective well-being among older adults. Considering the amygdala as a detector of relevance
(Sander et al., 2003), the findings of this study would be consistent with the hypothesis that older adults higher in subjective well-being may find negative information less salient than positive, resulting in less connectivity within an amygdala-mediated emotion-processing network in response to negative than to positive information. This difference in the salience of positive, as compared with negative, information among a subset of older adults would be consistent with behavioral findings in the literature-for example, that some older adults are more likely to remember positive rather than negative images (Mather \& Knight, 2005) or to bias gaze away from angry faces and toward happy ones (Isaacowitz, Toner, \& Neupert, 2009).

Critically, only a subset of the older adults - those higher in life satisfaction - showed differences in neural connectivity consistent with the positivity effect. Adults lower in life satisfaction did not show this valence effect, suggesting that they may not differ in their perceptions of the salience of positive and negative stimuli. It would be useful to test whether differences in emotional well-being among older adults are associated with perceptions of the relevance of positive and negative information in making important life choices in areas related to health, finances, and living situations.

An open question with regard to the positivity effect is to what extent it represents changes in older adults' processing of positive information (e.g., a focus on the positive), changes in their processing of negative information (e.g., an avoidance of the negative), or a combination of these factors (discussed by Murphy \& Isaacowitz, 2008). The results of the present study reveal the importance of considering both activity levels and connectivity profiles when examining this question at a neural level. When activity levels were considered, self-reported life satisfaction was found to moderate activity in the OFC and vmPFC only for the processing of positive information: higher life satisfaction was associated with lower activation during the encoding of positive images. Because this activity was measured for items that were successfully encoded into memory (i.e., for items that were later recognized), the lower activation levels may reflect enhanced neural efficiency. Reductions in neural activity are often shown on tasks that measure repetition priming (reviewed by Schacter, Dobbins, \& Schnyer, 2004), and so this pattern may reflect the fact that high-satisfaction individuals are able to encode positive items into memory in a more efficient fashion than are low-satisfaction individuals, without needing to activate the PFC to the same degree.

If only activity levels had been measured, it would have been tempting to conclude that life satisfaction was linked to the processing of positive but not negative information and that the effects were constrained to regions of the PFC. Yet the correlation matrices paint a different picture, with 
high-satisfaction individuals showing reliably lower correlations among multiple regions of the emotional memory network than did low-satisfaction individuals during the processing of negative images. Thus, at least in the present study, there is evidence that self-reported satisfaction is linked with the neural processing of both positive and negative information and that these connections are not circumscribed to the PFC. The SEM results further emphasize that self-reported satisfaction is not only linked to the recruitment of PFC regions; rather, the way that the amygdala communicates with other regions during the encoding of positive versus negative information is also related to life satisfaction. These findings emphasize that patterns of neural connectivity do not always parallel patterns of neural activity and underscore the importance for research to include both of these approaches.

The study has limitations that may inform future research. Perhaps the most important limitation is the inability to distinguish direction of influence between neural recruitment and life satisfaction, since they were measured concurrently. We cannot know from this study whether those individuals who are more satisfied with life are more likely to recruit specific neural pathways, or whether the recruitment of these pathways leads to greater life satisfaction. Multiple assessments would help determine the extent to which changes in one variable predict future changes in the other, as well as the stability of both variables over time.

The absence of a valence effect in patterns of brain activation among those low in life satisfaction could be the result of insufficient power to detect existing differences, and it would be important to replicate this study in a larger sample. As more sophisticated techniques for using SEM analyses with fMRI data are validated, it will be important to examine whether there is a specific point along the continuum of life satisfaction scores at which amygdala connectivity suddenly diverges as a function of valence, or whether the connectivity changes are more gradual. The two groups that are the focus of analyses in this study - the high- and low-satisfaction groups - are similar in a number of respects, including age, intelligence, memory, and executive functioning. There is also some evidence that their recent health was equivalent across the two groups. There are, however, other ways in which these two groups may differ that may contribute in unknown ways to the differing patterns of results found in this study. For example, Gerstorf et al. (2008) note that the age sensitivity of corticostriatal connections and dopamine-dependent processes implicated in reward processing may relate to late-life declines in well-being, and other recent research suggests that well-being declines with approaching death (e.g., Mroczek \& Spiro, 2005; Palgi \& Shmotkin, 2010). We continue to follow the participants in this study and, at a later time, will be able to examine whether proximity to death is linked with differences in life satisfaction and with neural processing of emotionally salient information.

Building on previous research (Addis \& McAndrews, 2006; Gilboa, 2004), this study demonstrates the potential of SEM to assess connectivity across brain regions. The application of SEM to connectivity data, however, is still new, and the relatively small samples typically used in fMRI studies challenge the limits of what is commonly thought to be a sufficient sample size (Kline, 2010). In this study, our conclusions are not based on the SEM analyses alone but are based on the combination of results revealed through the activity profiles, the correlation matrices of connectivity, and the SEM analyses of connectivity. All of these analytic approaches point to important differences between the low- and high-satisfaction groups.

This study addresses individual differences within a cohort of older adults. As such, it does not inform our understanding of possible differences within other age groups or whether these differences are stable over time. Additional longitudinal data will be required to determine whether the individual differences in neural activity and connectivity identified in this study are related to the observed positivity effect as people age. The results of this study, however, point to a potential mechanism that can be the focus of further longitudinal research on this question.

Acknowledgements We wish to thank Donna Addis and Ehri Ryu for their advice regarding connectivity methods, and an anonymous reviewer for drawing our attention to past research linking proximity to death and life satisfaction.

\section{References}

Addis, D. R., \& M. P. McAndrews (2006). Prefrontal and hippocampal contributions to the generation and binding of semantic associations during successful encoding. Neuroimage, 33(4), 1194-1206.

Addis, D., Leclerc, C., Muscatell, K., \& Kensinger, E. (2010). There are age-related changes in neural connectivity during the encoding of positive, but not negative, information. Cortex, 46, 425-433.

Benyamini, Y., Idler, E. L., Leventhal, H., \& Leventhal, E. A. (2000). Positive affect and function as influences on self-assessments of health: expanding our view beyond illness and disability. The Journals of Gerontology. Series B: Psychological Sciences and Social Sciences, 55, 107-116.

Blair, J. R., \& Spreen, O. (1989). Predicting premorbid IQ: a revision of the National Adult Reading Test. The Clinical Neuropsychologist, 3, 129-136.

Blais, M. R., Vallerand, R. J., Pelletier, L. G., \& Briere, N. M. (1989). French-Canadian validation of the satisfaction with life scale. Canadian Journal of Behavioral Science, 21, 210-223.

Carstensen, L., \& Mikels, J. A. (2005). At the intersection of emotion and cognition: aging and the positivity effect. Current Directions in Psychological Science, 14, 117-121.

Carstensen, L., Pasupathi, M., Mayr, U., \& Nesselroade, J. R. (2000). Emotional experience in everyday life across the adult life span. Journal of Personality and Social Psychology, 79, 644-655. 
Chang, E. C., Watkins, A. F., \& Banks, K. H. (2004). How adaptive and maladaptive perfectionism relate to positive and negative psychological functioning: testing a stress-mediation model in black and white college students. Journal of Counseling Psychology, 51, 93-102.

Charles, S., \& Carstensen, L. (2010). Social and emotional aging. Annual Review of Psychology, 61, 383-409.

Cipolotti, L., \& Bird, C. M. (2006). Amnesia and the hippocampus. Current Opinion in Neurology, 19, 593-598.

Cooney, R. E., Joorman, J., Atlas, L. Y., Eugene, F., \& Gotlib, I. H. (2007). Remembering the good times: neural correlates of affect regulation. Neuroreport, 18, 1771-1774.

Dale, A. M. (1999). Optimal experimental design for event-related fMRI. Human Brain Mapping, 8(2-3), 109-114.

Davis, M., \& Whalen, P. J. (2001). The amygdala: vigilance and emotion. Molecular Psychiatry, 6(1), 13-34.

Diener, E., Emmons, R. A., Larsen, R. J., \& Griffin, S. (1985). The satisfaction with life scale. Journal of Personality Assessment, 49, 71-75.

Gerstorf, D., Ram, N., Estabrook, R., Schupp, J., Wagner, G. G., \& Lindenberger, U. (2008). Life satisfaction shows terminal decline in old age: longitudinal evidence from the German SocioEconomic Panel Study (SOEP). Developmental Psychology, 44, $1148-1159$

Glueck, S., \& Glueck, E. (1968). Delinquents and nondelinquents in perspective. Cambridge: Harvard University Press.

Hamann, S. (2001). Cognitive and neural mechanisms of emotional memory. Trends in Cognitive Sciences, 5(9), 394-400.

Hamann, S. B., Ely, T. D., Hoffman, J. M., \& Kilts, C. D. (2002). Ecstasy and agony: activation of human amygdala in positive and negative emotion. Psychological Science, 13, 135-141.

Hartley, C. A., \& Phelps, E. A. (2010). Changing fear: the neurocircuity of emotion regulation. Neuropsychopharmacology, 35, 136-146.

Hawkley, L. C., \& Cacioppo, J. T. (2007). Aging and loneliness: downhill quickly? Current Directions in Psychological Science, 16, 187-191.

Heath, C. (1945). What people are. Cambridge: Harvard University Press.

Isaacowitz, D. M., Toner, K., \& Neupert, S. D. (2009). Use of gaze for real-time mood regulation: effects of age and attentional functioning. Psychology and Aging, 24, 989-994.

Joreskog, K., \& Sorbom, D. (1993). LISREL 8: users' reference guide. Chicago: Scientific Software International.

Kalisch, R., Wiech, K., Critchley, H. D., \& Dolan, R. J. (2006). Levels of appraisal: a medial prefrontal role in high-level appraisal of emotional material. Neuroimage, 30, 1458-1466.

Kashy, D. A., \& Snyder, D. K. (1995). Measurement and data analytic issues in couples research. Psychological Assessment, 7, 338-348.

Kjelstrup, K. G., Tuvnes, F. A., Steffenach, H. A., Murison, R., Moser E. I, \& Moser, M. B. (2002). Reduced fear expression after lesions of the ventral hippocampus. Proceedings of the National Academy of Sciences of the USA, 99(16), 10825-10830.

Kline, R. B. (2010). Principles and practice of structural equation modeling (3rd ed.). New York: Guilford.

Lang, P., Bradley, M., \& Cuthbert, B. (1999). International Affective Picture System (IAPS): technical manual and affective ratings. Gainesville: Center for Research in Psychophysiology.

Leclerc, C. M., \& Kensinger, E. A. (2008). Age-related differences in medial prefrontal activation in response to emotional images. Cognitive, Affective, \& Behavioral Neuroscience, 8, 153-164.

Löckenhoff, C. E., \& Carstensen, L. L. (2004). Socioemotional selectivity theory, aging, and health: the increasingly delicate balance between regulating emotions and making tough choices. Journal of Personality, 72, 1395-1424.
Mather, M., Canli, T., English, T., Whitfield, S., Wais, P., Ochsner, K., et al. (2004). Amygdala responses to emotionally valenced stimuli in older and younger adults. Psychological Science, 15, 259-263.

Mather, M., \& Carstensen, L. L. (2005). Aging and motivated cognition: the positivity effect in attention and memory. Trends in Cognitive Sciences, 9, 496-502.

Mather, M., \& Knight, M. (2005). Goal-directed memory: the role of cognitive control in older adults' emotional memory. Psychology and Aging, 20, 554-570.

McIntosh, A., \& Gonzalez-Lima, F. (1994). Network interactions among limbic cortices, basal forebrain, and cerebellum differentiate a tone conditioned as a Pavlovian excitor or inhibitor: fluorodeoxyglucose mapping and covariance structural modeling. Journal of Neurophysiology, 72(4), 1717-1733.

Morris, J. C., Heyman, A., Mohs, R. C., Hughes, J. P., van Belle, G., Fillenbaum, G., et al. (1989). The consortium to establish a registry for Alzheimer's disease (CERAD): PartI. Clinical and neuropsychological assessment of Alzheimer's disease. Archives of Neurology, 49, 1253-1258.

Mroczek, D. K., \& Kolarz, C. M. (1998). The effect of age on positive and negative affect: a developmental perspective on happiness. Journal of Personality and Social Psychology, 75, 1333-1349.

Mroczek, D. K., \& Spiro, A. (2005). Change in life satisfaction during adulthood: findings from the Veterans Affairs Normative Aging Study. Journal of Personality and Social Psychology, 88, 189202.

Murphy, N. A., \& Isaacowitz, D. M. (2008). Preferences for emotional information in older adults: a meta-analysis of memory and attention studies. Psychology and Aging, 23, 263-286.

Murty, V. P., Ritchey, M., Adcock, R. A., \& LaBar, K. S. (2011). Reprint of: fMRI studies of successful emotional memory encoding: a quantitative meta-analysis. Neuropsychologia, 49, 695-705.

Ochsner, K. N., Bunge, S. A., Gross, J. J., \& Gabrieli, J. D. E. (2002). Rethinking feelings: an fMRI study of the cognitive regulation of emotion. Journal of Cognitive Neuroscience, 14, 1215-1229.

Öhman, A., \& Mineka S. (2001). Fears, phobias, and preparedness: Toward an evolved module of fear and fear learning. Psychological Review, 108(3), 483-522.

Padmala, S., \& Pessoa, L. (2008). Affective learning enhances visual detection and responses in primary visual cortex. Journal of Neuroscience, 28, 6202-6210.

Palgi, Y., \& Shmotkin, D. (2010). The predicament of time near the end of life: time perspective trajectories of life satisfaction among the old-old. Aging \& Mental Health, 14, 577-586.

Palmer, B., Donaldson, C., \& Stough, C. (2002). Emotional intelligence and life satisfaction. Personality and Individual Differences, 33, 1091-1100.

Pavot, W., Diener, E., Colvin, C. R., \& Sandvik, E. (1991). Further validation of the satisfaction with life scale: evidence for the cross-method convergence of well-being measures. Journal of Personality Assessment, 57, 149-161.

Phelps, E. A., \& LeDoux, J. E. (2005). Contributions of the amygdala to emotion processing: from animal models to human behavior. Neuron, 48, 175-187.

Sander, D., Grafman, J., \& Zalla, T. (2003). The human amygdala: an evolved system for relevance detection. Reviews in the Neurosciences, 14, 303-316.

Schacter, D. L., Dobbins, I. G., \& Schnyer, D. (2004). Specificity of priming: a cognitive neuroscience perspective. Nature Reviews. Neuroscience, 5, 853-862.

Spaniol, J., Davidson, P. S., Kim, A. S., Han, H., Moscovitch, M., \& Grady, C. L. (2009). Event-related fMRI studies of episodic encoding and retrieval: meta-analyses using activation likelihood estimates. Neuropsychologia, 47, 1765-1779. 
St. Jacques, P. L., Bessette-Symons, B., \& Cabeza, R. (2009a). Functional neuroimaging studies of aging and emotion: fronto-amygdalar differences during emotional perception and episodic memory. Journal of the International Neuropsychological Society, 15, 819-825.

St. Jacques, P. L., Dolcos, F., \& Cabeza, R. (2009b). Effects of aging on functional connectivity of the amgydala during subsequent memory for negative pictures: a network analysis of fMRI data. Psychological Science, 20, 74-84.

Steiger, J. H. (1980). Tests for comparing elements in a correlation matrix. Psychological Bulletin, 87, 245-251.

Ungerleider, L. G., \& Haxby, J. V. (1994). 'What' and 'where' in the human brain. Current Opinion in Neurobiology, 4, 157-165.
Vaillant, G. E. (1977). Adaptation to life. Boston: Little, Brown.

Vertes, R. P. (2006). Interactions among the medial prefrontal cortex, hippocampus and midline thalamus in emotional and cognitive processing in the rat. Neuroscience, 142, 1-20.

Williams, L. M., Brown, K. J., Palmer, D., Liddell, B. J., Kemp, A. H., Olivieri, G., et al. (2006). The mellow years? Neural basis of improving emotional stability over age. The Journal of Neuroscience, 26, 6422-6430.

Wright, C. I., Wedig, M. M., Williams, D., Rauch, S. L., \& Albert, M. S. (2006). Novel fearful faces activate the amygdala in healthy young and elderly adults. Neurobiology of Aging, 27, 361-374. 